\title{
Rocket engine nozzle side load transient analysis methodology- a practical approach
}

\author{
John J. Shi*, Ph.D. \\ Boeing-Rocketdyne Division, Canoga Park, California, 91303, U.S.A.
}

\begin{abstract}
During the development stage, in order to design/to size the rocket engine components and to reduce the risks, the local dynamic environments as well as dynamic interface loads must be defined. There are two kinds of dynamic environment, i.e. shock transients and steady-state random and sinusoidal vibration environments. Usually, the steady-state random and sinusoidal vibration environments are scalable, but the shock environments are not scalable. In other words, based on similarities only random vibration environments can be defined for a new engine. The methodology covered in this paper provides a way to predict the shock environments and the dynamic loads for new engine systems and new engine components in the early stage of new engine development or engine nozzle modifications.
\end{abstract}

\section{Nomenclature}

$F_{\text {static }}(t)=$ Quasi static side loads

$F(t) \quad=$ Fluctuating asymmetric dynamic side loads

$F_{r m s}(i) \quad=$ The nns dynamic side loads

$p_{w} \quad=$ Nozzle wall free stream dynamic pressure

$p^{\prime} \quad=$ Nozzle wall boundary layer fluctuating dynamic pressure

$p_{r m s}^{\prime} \quad=$ The rms boundary layer fluctuating dynamic pressure

$p_{a} \quad=$ Ambient pressure

$P_{c} \quad=$ Chamber pressure

$f_{p} \quad=$ Symmetric side loads pressure pulse oscillating frequency

$p_{s}(t) \quad=$ Pressure pulses for symmetric side loads

$p_{\text {peak }}=$ Peak value for the pressure puises

\section{Introduction}

At sea level operations, all the rocket engines, especially for a highly over-expanded nozzle, will be excited by side loads, i.e. non-axial forces due to flow separations, during engine ignition and shutdown. Since rocket engines are designed to produce axial thrust to power the vehicles, it is not desirable to be subject to excitations by non-axial static and/or dynamic input forcing functions. In the past, several engine failures were attributed to the side loads. The J-2S engine had excessive side loads such that the gimbal block retaining bolts failed in tension. The Space Shuttle Main Engine (SSME) had the side load induced 'steerhorn', i.e. a component of the liquid hyrogen $\left(\mathrm{LH}_{2}\right)$ feedline, low cycle fatigue cracks that damaged the nozzle [1]. More recently the European Vulcain engine and Japanese LE-7A engine, i.e. the first stage main engine of the H-IIA launch vehicle, also had problems due to nozzle side loads. In order to resolve side loads issues on Vulcain, the Europeans created a very thorough experimental and analytical program to understand the nozzle flow physics that induce side loads[2]. The LE-7A engine nozzle side loads were severe enough to fail the engine actuators and cause the regenerative cooling tubes to rupture [3]. Since side loads can cause system level failures, e.g. gimbal bearing, actuator, and local failures, e.g. the "steerhorn", cooling tubes, they are considered to be a risk item or a design issue for a new rocket engine development.

For structural dynamics, two types of analysis have been performed to simulate the nozzle side load transients. The

*Associate technical fellow, Structural Dynamics/IB31, AIAA member 
first is the large asymmetrical input forcing functions used to simulate the side loads caused by flow separation existing over a large portion of the nozzle due to low chamber pressure. The second type is the symmetrical input forcing functions used to simulate the loading which occurs as the shock passes through the nozzle exit plane. The shock will pulse in and out of the end of the nozzle for a few oscillations. This results in a circumferential loading over the last part of the nozzle. Both types of loading must be accounted for during development. Since the asymmetrical input forcing functions are in the frequency range below $50 \mathrm{~Hz}$, they will excite the entire engine system. The symmetrical ones are in the higher frequency range, and based on the Space Shuttle Main Engine(SSME) experience, they are above $100 \mathrm{~Hz}$. Therefore, these forcing functions will mainly excite the local

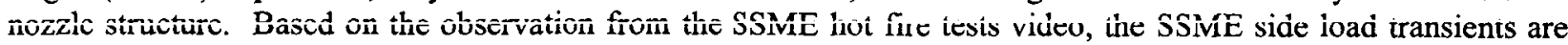
depicted graphically in Fig. 1.

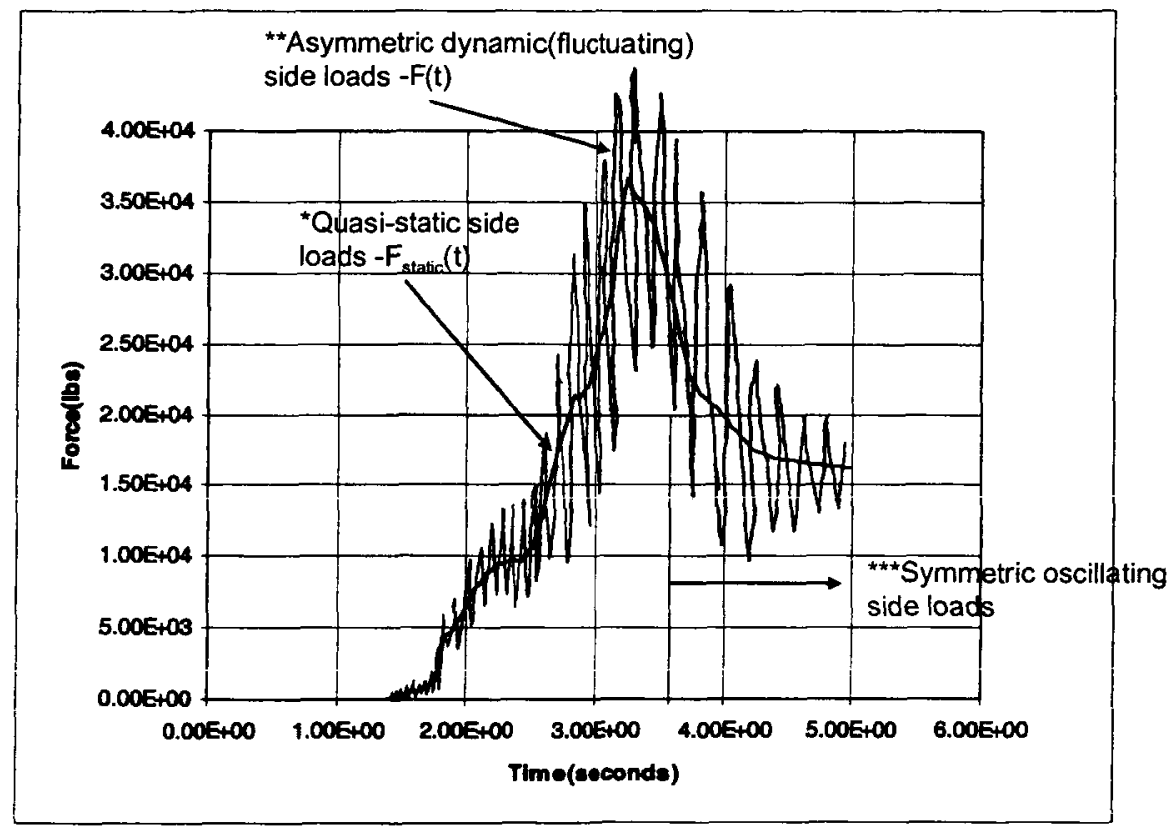

*Due to dynamic pressure of the free stream, i.e. $\mathrm{p}_{\mathrm{w}}$

** Due to fluctuating dynamic pressure in the boundary layer, i.e. p'

*** Due to fluid/structure interactions

Fig. 1 SSME side loads scenario

According to Fig.1, the quasi-static side loads and the dynamic side loads will act simultaneously. The quasi-static side loads are due to dynamic pressure of the free stream flow, i.e. the $\mathrm{p}_{\mathrm{w}}$. The asymmetrical dynamic side loads are due to fluctuating dynamic pressure in the boundary layer, i.e. the p', while the symmetric side loads are due to vortex fluctuations and fluid/structure interaction, e.g. flutter. Based on available literature, it is a novel idea to introduce the p' into the mechanism of the side load transients. The J-2S engine hot fire data [4] were used to derive the input forcing functions for the asymmetrical dynamic side loads. The SSME subscale nozzle air flow test measurements were used to develop the input forcing functions for the symmetric dynamic side loads. By applying the forcing functions on the SSME block II engine system finite element model, the 3- $\sigma$ peak dynamic loads for engine components were calculated. Moreover, the component dynamic loads derived from the SSME hot fire test measurements were used to validate analytically predicted dynamic loads.

This paper describes the work done at Boeing to simulate the nozzle side load transients for the analysis of the rocket engine systems developed at the Rocketdyne division. The primary purpose of this analysis is to predict the 
dynamic environments and the dynamic loads for the engine components in the early stage of new engine development or engine nozzle modification. The methodology and the analysis process were developed to derive the input forcing functions for both asymmetric and symmetric dynamic load transients as shown in Fig. 1.

\section{Asymmetric dynamic side loads}

The asymmetric dynamic side loads were due to free shock separation (FSS) in the nozzles, where the separated flow continues as a free jet, see Fig. 2.

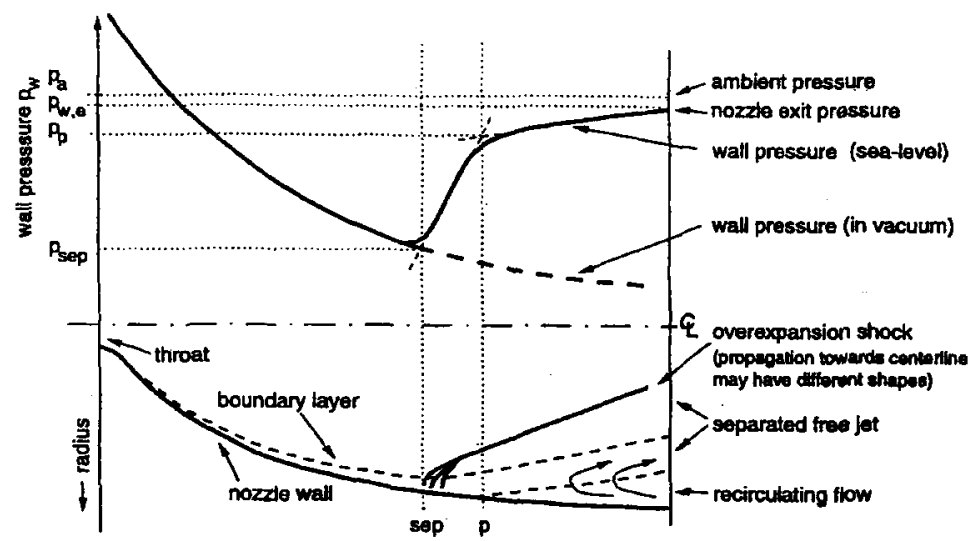

Fig. 2 Free shock separation (FSS)

Flow separation in the rocket nozzles occurs whenever the wall pressure $p_{w}$ is much lower than ambient pressure $p_{a}$. According to J-2S test measurements [4], the flow separation occurs randomly when $1 / 4 \leq p_{w} / p_{a} \leq 1 / 2$. Based on this assumption, a methodology called the "skew plane" separation was developed by the Aerodynamic Process at Rocketdyne to determine the quasi-static side loads $\left(\mathrm{F}_{\text {static }}\right.$ ) as shown in Fig. 3. According to Fig. 3, the nozzle flow will be full, when $p_{w} / p_{a}>1 / 2$, and the nozzle flow separation occurs randomly when $1 / 4 \leq p_{w} / p_{a} \leq 1 / 2$. Moreover, maximum quasi-static side load occurs when $p_{w} / p_{a}=1 / 4$ occurs at the nozzle exit.

Due to randomness of the flow separation, the skew plane will change with time and the flow inside the nozzle is unsymmetrical. The resultant, i.e. the integration, of the unsymmetrical distribution of the $p_{w}$ will cause the quasistatic side loads, while the resultant of the $p$ ' will cause the fluctuating dynamic side loads. $F_{\text {static }}(t)$ is used to represent the quasi-static side loads, and the dynamic side loads are represented by $F(t)$. According to Ref. [4], the dynamic side loads, i.e. $F(t)$, were a random function that had a relatively flat spectrum in the frequency range 5 to $50 \mathrm{~Hz}$. As shown in Fig. 1, $F_{\text {static }}(t)$ and $F(t)$ will act simultaneously. The relationship between $F(t)$ and $F_{\text {static }}(t)$ is $F_{\text {rns }}(t) / F_{\text {static }}(t)$ is a constant. This is based on the relationship between the $p$ ' and the $p_{w}$. According to Ref. [5], the ratio $\mathrm{p}_{\text {rms }}^{\prime} / \mathrm{p}_{\mathrm{w}}$ is a constant which is called the scaling factor in this paper.

Based on the technical background discussed above, an analysis process, see Fig. 4, has been developed to perform asymmetric side load transient analysis. Since there are an unlimited number of solutions for any random process, the methodology shown in Fig. 4 is an approach to generate side load transients randomly in order to excite an engine system model. Moreover, the Monte Carlo process was used to generate random orientation for each side load transient.

As indicated in Fig. 4, there are two basic inputs required for the side load transient analysis. The first one is the chamber pressure $\left(\mathrm{P}_{\mathrm{c}}\right)$ time history during engine start and cutoff. The second one is the quasi-static side loads as a function of the $\mathrm{P}_{c}$. Both inputs can be analytically derived or obtained from actual test data. The scaling factor will be determined empirically. 


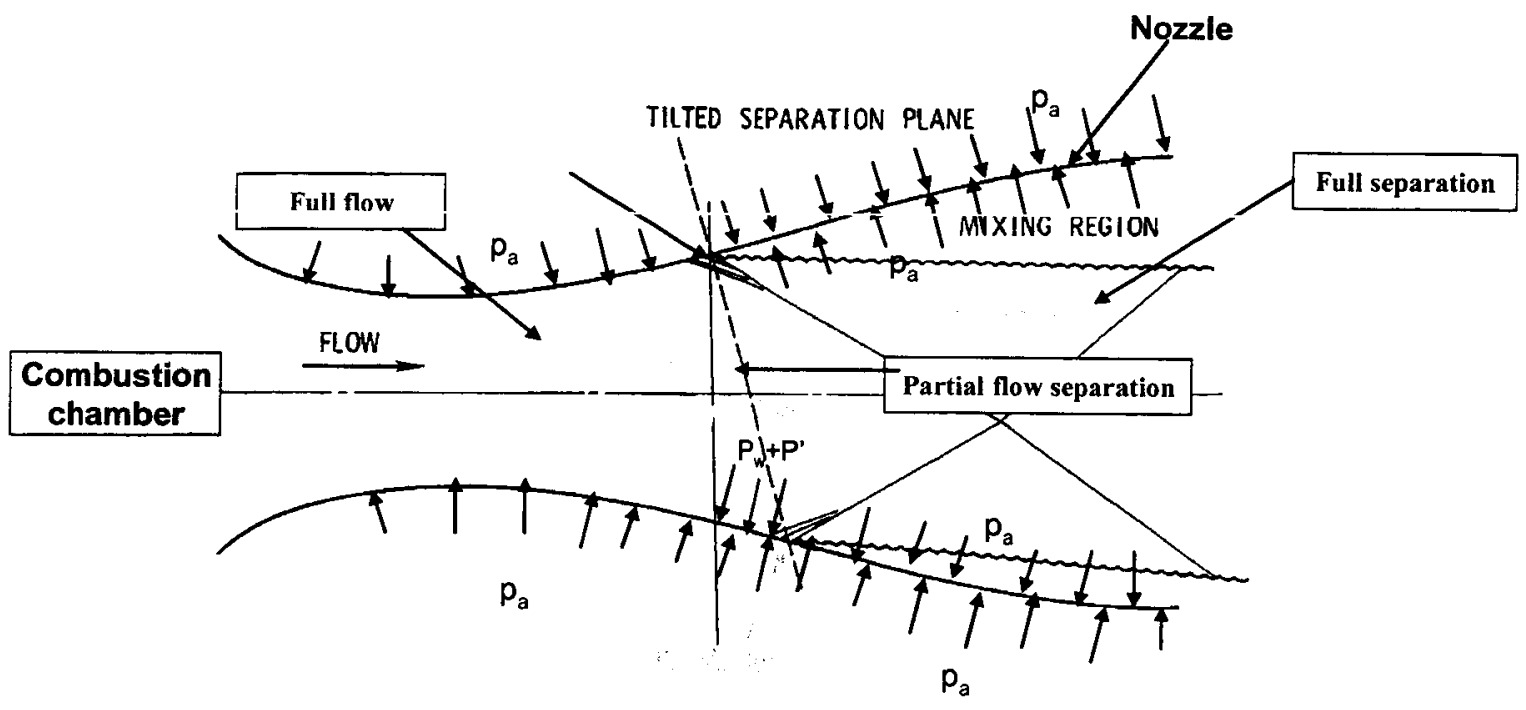

Figure 39. Side Load Tilted Separation Plane

Fig.3 Skew plane separation, $p_{\mathrm{a}}=14 \mathrm{psi}$

\section{Engine system nozzle asymmetric side loads analysis process}

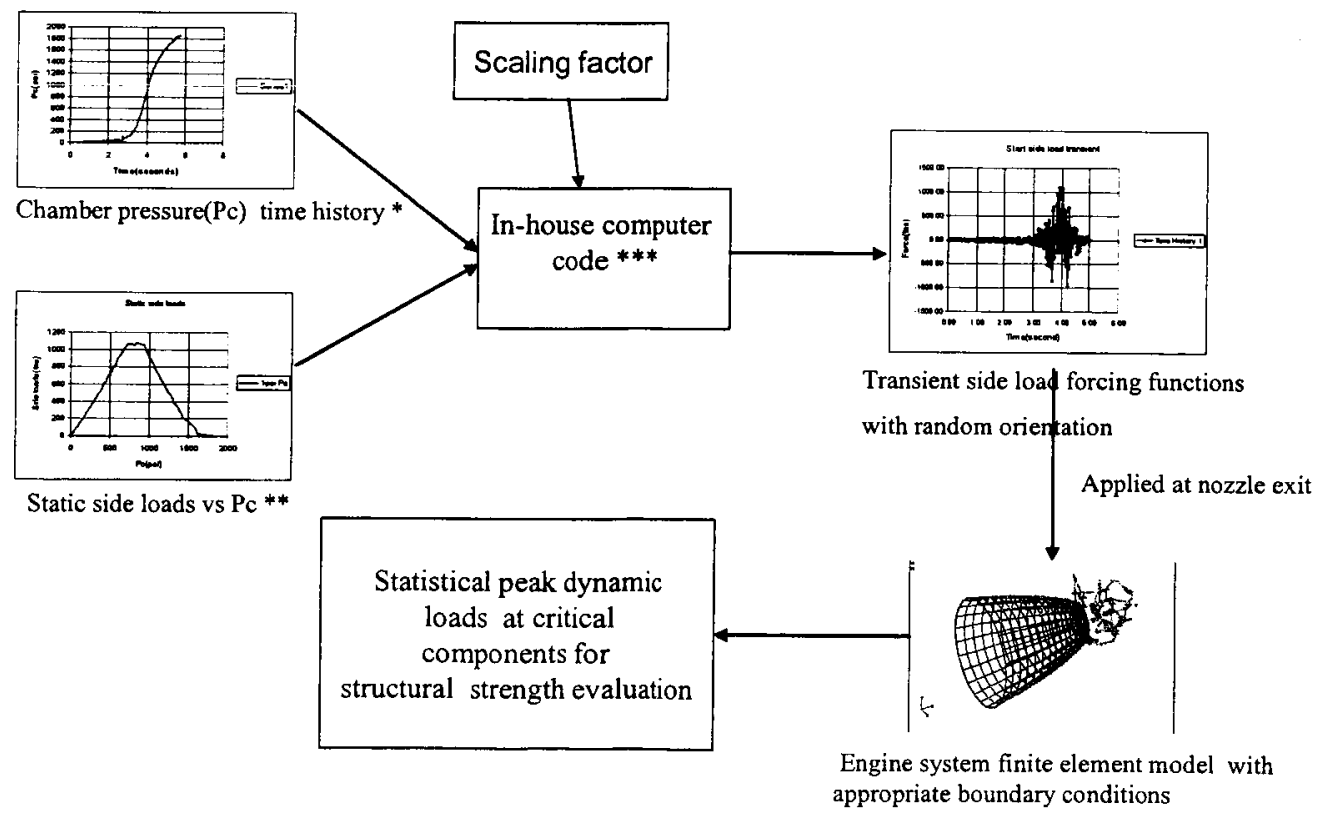

Fig. 4 Asymmetric side load analysis process 


\section{Symmetric dynamic side loads}

The symmetric dynamic side loads were due to restricted shock separation (RSS) in the nozzle, where the separated flow reattaches to the nozzle wall, thereby forming a closed recirculation bubble, see Fig. 5. According to [4], it appears that the separation is symmetrical, and no apparent lateral forces were observed. It is obvious when the flow is symmetrical, there will be no resultant forces in the lateral direction but the individual force, i.e. $F_{s}(t)$ in Fig. 8, may be large. Based on the subscale air test results from Ref. [1], the loadings on the nozzle are pulses that varied axialiy and circumferentially. The pressure fiuctuation is uniform circunferenialily, bui it is in phase and varies in amplitude axially. The amplitude of the pulse initially increases moving toward the throat and then decreases to a very small values. The description of the idealized pulses is shown in Fig. 6.

As shown in Fig. 6, the period for the pressure pulses is T seconds. Therefore, the oscillating frequency $\left(f_{p}\right)$ for the pulses is $1 / \mathrm{T} \mathrm{Hz}$. The oscillating frequency will be determined empirically by tests or analytically calculated by the CFD models. Moreover, the spatial distributions of the pressure pulses on the nozzle will be modulated by the fundamental mode of the nozzle. The reason for the modulation is due to fluid/structure interaction, but it is not very clear what triggers the interaction. Based on the author's judgment, it is due to the flutter that causes the structure, i.e. the nozzle, to be excited and then locked into the fundamental mode of the nozzle, i.e. the $N=2$ mode as shown in Fig. 7. Therefore, it is a very complicated phenomenon with the oscillating pressure pulses created by vortex fluctuations and then synchronized with the fundamental mode of the nozzle. The vortex fluctuations are similar to the fluctuation of the Kármán vortices with a frequency of $\mathrm{f}_{\mathrm{p}} \mathrm{Hz}$.

Moreover, when the nozzle is excited by the $\mathrm{N}=2$ mode, it is deformed from a circle to an ellipse. Therefore, instead of four equal quadrants, two (quadrants 1 and 3), are contracted and the other two(quadrants 2 and 4) are expanded as shown in Fig. 7. When the quadrant is contracted, the volume inside the circulation bubble is reduced. When the quadrant is expanded, the volume is increased. According to the perfect gas law, at the time when the nozzle is deformed as shown in Fig. 7, quadrants 1 and 3 will have higher pressure, while quadrants 2 and 4 will have lower pressure. By assuming the wall pressure in quadrants 2 and 4 to be lower than ambient pressure, the flow in quadrants 2 and 4 will be separated from the wall again with zero gage pressure, i.e. $\Delta p$. The gage pressure distribution around the nozzle is shown in Fig. 8. Since the nozzle is vibrated in the $\mathrm{N}=\mathbf{2}$ mode with a natural frequency to be $f_{s}$, in every half cycle or half period of vibration the pressure distributions will be switched between quadrants until the pressure pulses stop. The $f_{s}$ will be determined analytically by finite element model or empirically by modal testing. As for the amplitude of the pulses, i.e. the $p_{\text {peak }}$ as shown in Fig. 6 , it should be determined empirically by tests if feasible. In this paper, an analytical approach was developed to estimate the $p_{p e a k}$ as discussed below.

By definition, $F_{s}(t)$ is the total force due to the pressure, i.e. $p_{s}(t)$, in the entire area of one quadrant as shown in Fig. 8. As discussed at the beginning of this section, since the $p_{s}(t)$ is uniform circumferentially but varied from the nozzle exit to the throat, the $F_{s}(t)$ become a linear function of the $p_{\text {peak }}$. Moreover, according to Fig. 1 the symmetrical oscillating side loads will occur shortly after the quasi-static side loads reach the peak value. Therefore, it may be reasonable to use the peak value of quasi-static side loads, i.e. $F_{\text {static, } \max }$ to derive the $p_{\text {peak }}$. This is a practical approach, because the quasi-static side loads can be determined analytically using the "skew plane" method as shown in Fig. 3.

The methodology discussed above will be used to derive input forcing functions to excite the engine system model later in this paper. The analysis results will be used to validate or substantiate the assumptions. 


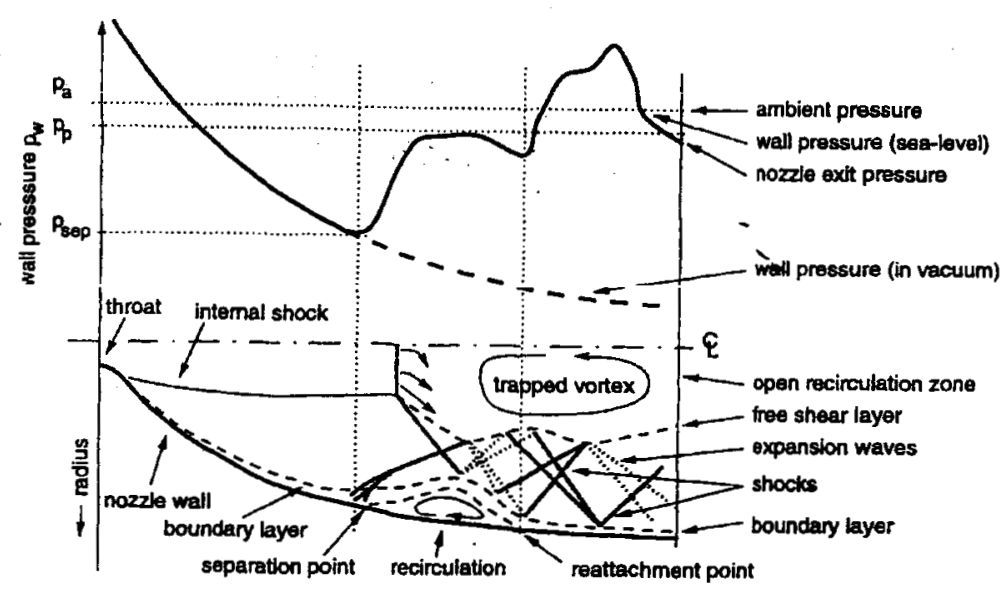

Fig. 5 Restricted shock separation (RSS)

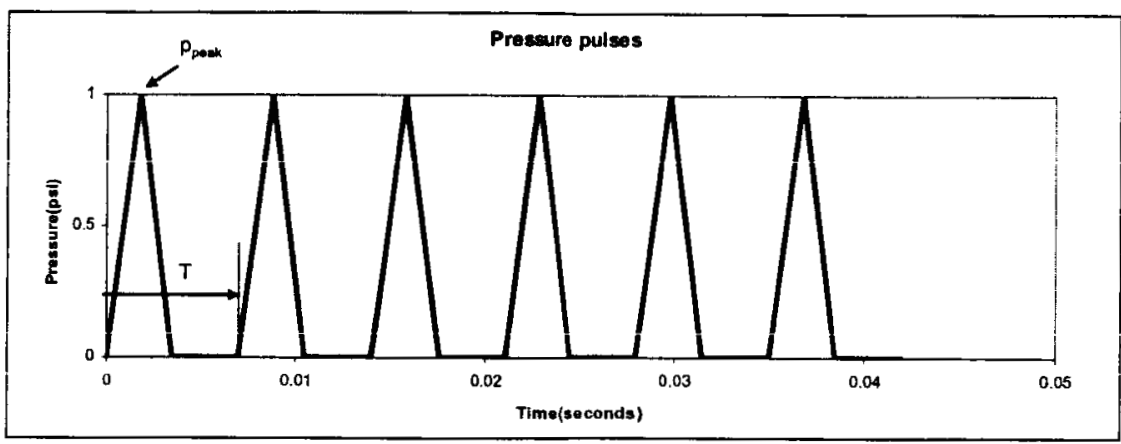

Fig 6 Pressure pulses during period of jet oscillations, $p_{s}(t)$

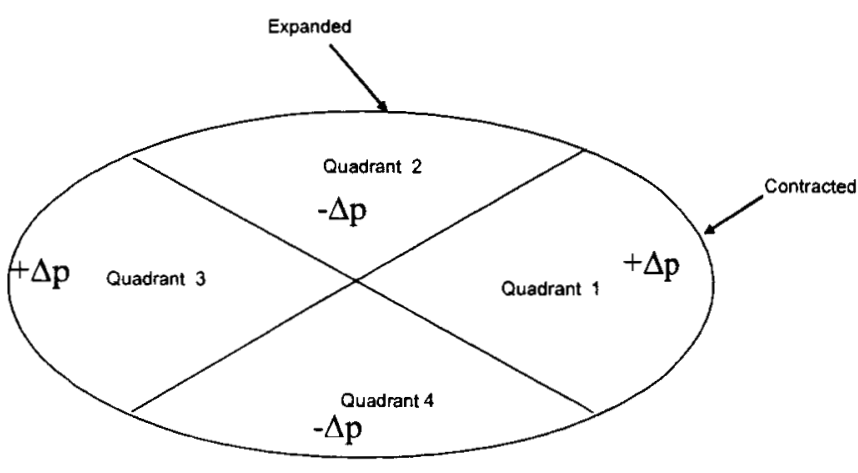

Fig. $7 \mathrm{~N}=2$ mode

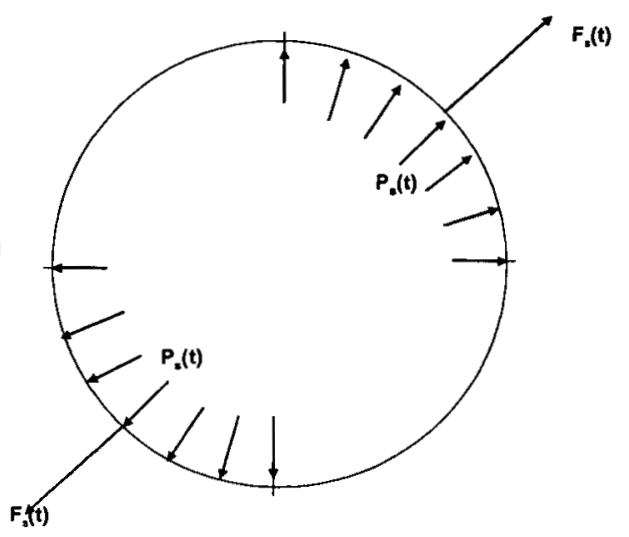

$F_{s}(t)=\int p_{s}(t) d A$ in one quadrant

Fig. 8 Gage pressure $(\Delta p)$ distribution 


\section{Application}

Due to availability of extensive hot fire test measurements for the SSME engine system, analyses will be performed on the SSME finite element model to validate the methodology presented in this paper. It will be used to derive the scaling factor, see Fig. 4, for the asymmetric side load analysis. It also will be used to validate/substantiate the assumptions for the symmetric side load analysis.

\section{SSME engine system finite element model}

A SSME engine system structural dynamic finite element model (FEM) was provided by the SSME Structural Dynamics unit. It is a model for the block II engine system as shown in Fig. 10.

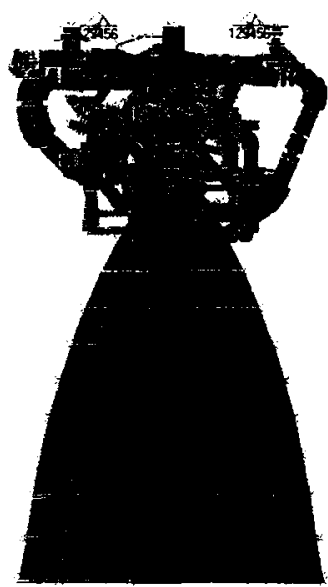

Fig.9 SSME engine system FEM

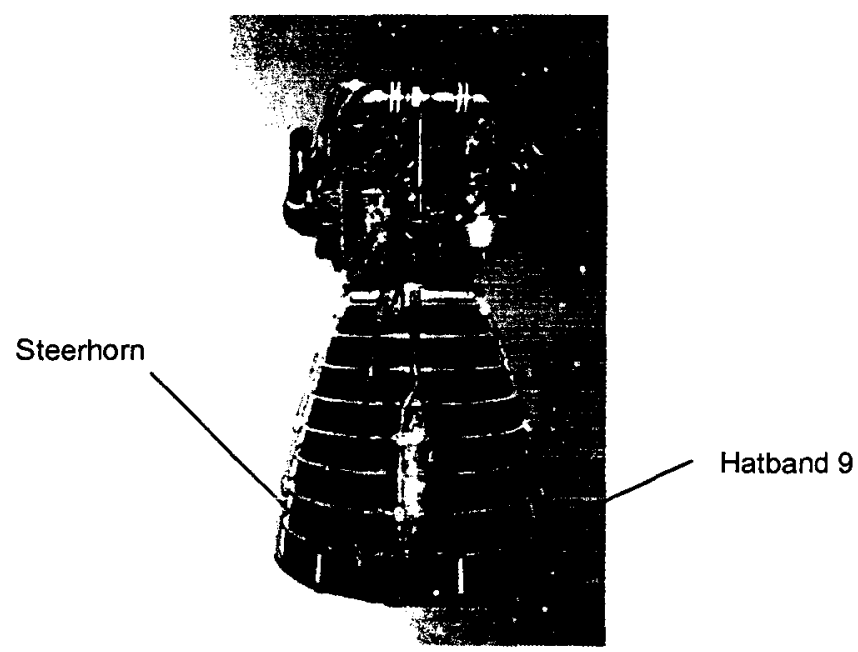

Fig. 10 SSMF engine system

In the nozzle area, all the hatbands including the aft manifold are modeled as beams. The boundary conditions are pinned at the gimbal and fixed at the actuators. The actuators are modeled as tension-compression beams.

By performing natural frequency analysis on the model, two engine system pendulum modes were identified to be about $7 \mathrm{~Hz}$. As for the nozzle shell modes, they are listed below.

\begin{tabular}{|c|c|}
\hline \# of harmonics (N) & Frequency $(\mathrm{Hz})$ \\
\hline 2 & 23 \\
\hline 3 & 56 \\
\hline 4 & 94 \\
\hline
\end{tabular}

\section{Asymmetric side load input forcing functions}

During the engine thrust buildup, the combustion chamber pressure increases rapidly from ambient to maximum pressure at full power operation as shown in Fig. 11. The data shown in Fig. 11 are the measured data for a SSME hot fire test. The test number is Block II test 9010929 . Within about 5 seconds, the $P_{c}$ increases from ambient to about 2700 psi.

By using the "skew plane" separation method, the quasi-static side loads vs the $\mathrm{P}_{\mathrm{c}}$ are derived by the Aerodynamics analyst as shown in Fig.12. Based on data shown in Fig.6, the maximum static side load is about 38,000 lbs at $p_{c}=$ $1,750 \mathrm{psi}$, i.e. pressure ratio $\left(\mathrm{p}_{\mathrm{c}} / \mathrm{p}_{\mathrm{a}}\right) \sim 120$. 


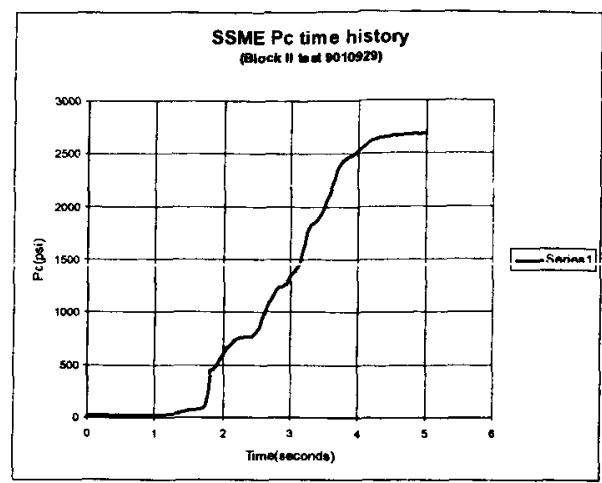

Fig. 11 SSME start transient

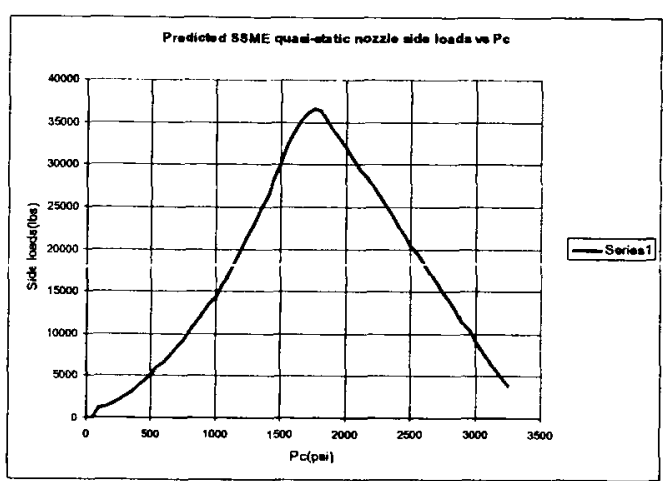

Fig. 12 Predicted SSME quasi-static side loads

By using data in Fig. 11 and Fig. 12 as inputs into the analysis process, see Fig. 4, the in-house computer code was used to generate 100 side load transients randomly. As examples, 2 side load time histories are shown in Figs.13. Each case also has a random orientation in terms of an angle assigned to it.
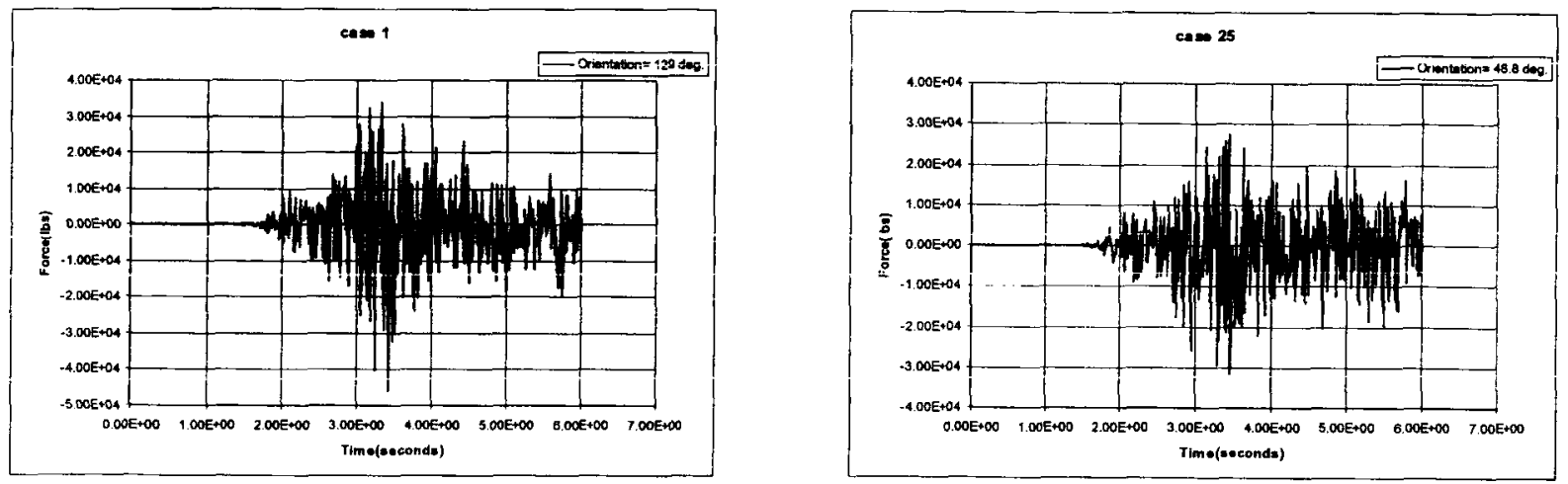

Fig. 13 Side load transients

\section{Symmetric side load input forcing functions}

On two occasions in 1979 during the SSME development hot-firing program, a component (called the steerhorn due to its shape) of the nozzle coolant, i.e. liquid hydrogen $\left(\mathrm{LH}_{2}\right)$, supply line failed during the cutoff transient. The failures were attributed to high symmetric side loads. In order to characterize the symmetric side load transient, i.e. pulse frequency, amplitude, and number, air flow tests were conducted on a 1/9 scale model of the SSME nozzle [1]. Based on data presented in the paper [1] and some un-published documents, a set of the symmetric side loads for the SSME was constructed as shown in Fig. 14.

According to Fig. 14, the frequency $\left(f_{p}\right)$ for the pulses is $\sim 140 \mathrm{~Hz}$, i.e. the period $=.007$ seconds. The pulses are distributed axially from the aft manifold to the $7^{\text {th }}$ hatbnand. The variations of the amplitude ( $p_{\text {peak }}$ ) along the nozzle axis are shown in Fig. 14. By integrating the pressure from the aft manifold to the $7^{\text {th }}$ hatband in one quadrant the resultant force $\mathrm{F}$ is $37,000 \mathrm{lbs}$, which is the same as the maximum quasi-static side load as shown in Fig.1. Moreover, the pulses will be modulated by the frequency $(\sim 25 \mathrm{~Hz})$ of the $\mathrm{N}=2$ mode. 

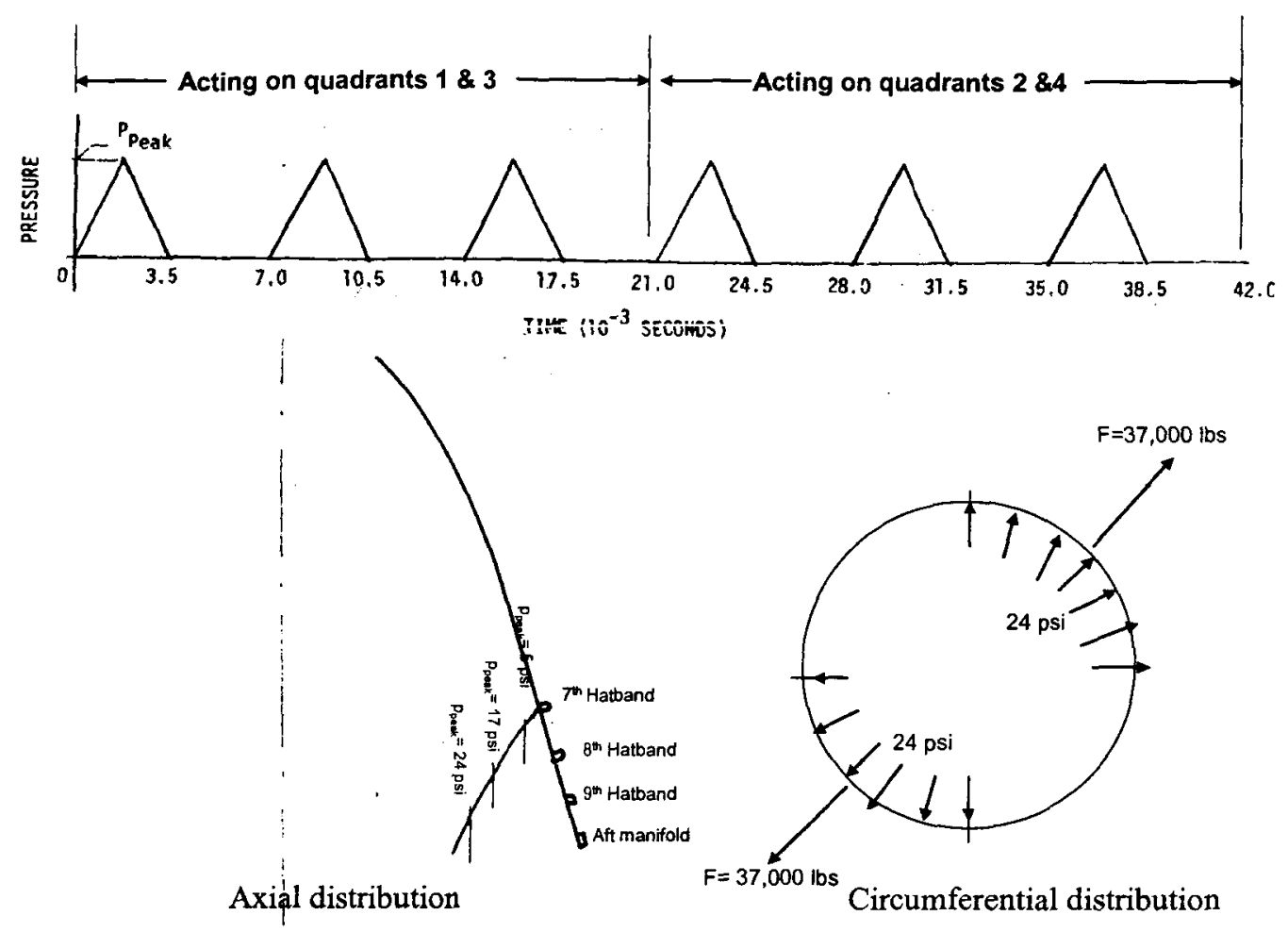

Fig. 14 SSME symmetric pressure pulses

\section{$\underline{\text { Results }}$}

The analysis process for the asymmetrical dynamic side load transient is shown in Fig. 4. One by one the 100 randomly generated cases as shown in Fig. 13 were applied at the nozzle to excite the SSME engine system finite element model, see Fig. 9. The force application locations, i.e. from the nozzle exit to the pressure center, and the orientation of the loading were randomly generated by the in-house computer code. The pressure center is about 40 inches from the exit. An automated analysis process was developed to run any number of cases, e.g. 100 cases or more, efficiently. The results from the 100 cases were processed to obtain the statistical dynamic loads in terms of the mean and the standard deviation $(1 \sigma)$ values at any locations in the engine system.

As for the symmetric side load transient analysis, the input forcing function shown in Fig. 14 was applied on the SSME nozzle at the specified locations as shown in the figure. The pressure loadings were distributed at appropriate locations, i.e. the quadrants and the axial locations from the aft manifold to the $7^{\text {th }}$ hatband, and at the appropriate time as shown in Fig. 14. It is a very complicated analysis procedure. After performing the response analysis, the peak dynamic loads were generated for any locations in the engine system.

\section{Discussions}

In order to validate the methodology, the SSME hot-fire measurements were obtained. Even though the SSME has a very extensive hot-fire measurements data base, transient load measurements are very limited. The SSME engines had strain gage measurements on the hatbands $6,7,8$ and 9 as well as force measurements on the actuators. The hatband strain measurements were converted to the bending moments. There were 87 data points to determine the $3 \sigma$ dynamic bending moments during the engine start and cutoff for hatband 6 through 9 . As for the actuator loads there were 122 data points to determine the $3 \sigma$ dynamic actuator loads. 
In order to correlate with the analysis results, the SSME hot-fire measurements at the hatbands and the actuators were retrieved from the stress reports. The analysis results along with the test measurements are summarized in Table 1.

Table 1 Test/analysis correlation

\begin{tabular}{|c|c|c|c|c|c|}
\hline $\begin{array}{l}\text { Hatband \# or } \\
\text { actuator }\end{array}$ & $\begin{array}{l}3 \sigma^{\circ} \text { peak loads } \\
\text { based on hot-fire } \\
\text { measurements }\end{array}$ & $\begin{array}{l}\text { Predicted } 3 \sigma \text { peak } \\
\text { loads } \\
\text { due to asymmetric } \\
\text { side loads } \\
\text { (Scaling factor= .13) }\end{array}$ & $\begin{array}{l}\text { Predicted peak } \\
\text { loads } \\
\text { due to symmetric } \\
\text { side loads }\end{array}$ & $\begin{array}{l}\text { Predicted total } \\
\text { peak loads** }\end{array}$ & $\begin{array}{l}\text { Predicted loads/ } \\
\text { measured loads }\end{array}$ \\
\hline 6 & 17,000 in-lbs & 6,400 in-lbs & 19,200 in-lbs & 20,240 in-lbs & 1.19 \\
\hline 7 & 25,100 in-lbs & 10,300 in-lbs & 30,700 in-Jbs & 32,380 in-lbs & 1.29 \\
\hline 8 & 34,400 in-lbs & 13,900 in-lbs & 41,000 in-lbs & 43,290 in-lbs & 1.26 \\
\hline 9 & 51,700 in-lbs & 16,900 in-lbs & 51,800 in-lbs & 54,490 in-lbs & 1.05 \\
\hline Actuator & $64,000 \mathrm{lbs}$ & $64,000 \mathrm{lbs}$ & $2,300 \mathrm{lbs}$ & $64,040 \mathrm{lbs}$ & 1.00 \\
\hline
\end{tabular}

*87 samples for the hat bands and 122 samples for the actuators

**SRSS the loads due to asymmetric and symmetric side loads

The correlation between the analysis and the test results is excellent. As expected, the asymmetric side loads will excite the engine system, i.e. high actuator loads but low hatband loads, while the symmetric side loads will excite the nozzle local structure, i.e. low actuator loads but high hatband loads. Based on the above reason, only the actuator loads were used to derive the scaling factor for the asymmetric side loads. The scaling factor was determined to be .13. Furthermore, the SRSS( Square Root of the Sum Of Squares) method was used to combine the loads due to asymmetric and symmetric side loads, because they are uncorrelated and occur in different frequency ranges.

Since both the asymmetric and the symmetric side loads are random processes, it is possible that the actual loads are higher than those given in Table 1. For some asymmetric side load transient cases, the dynamic loads may be higher than $3 \sigma$. Parametric studies also showed that the slower the thrust build-up, the higher the actuator dynamic loads due to the asymmetric side loads. For the symmetric side loads, the peak dynamic loads will be higher than the levels shown in Table 1, if more pressure pulses, i.e. $>6$ pulses, were generated by vortex fluctuations. Studies also showed the nozzle hatband bending moments were sensitive to $\mathrm{N}=2$ mode modulation.

Moreover, according to Fig. 1, the dynamic loads due to dynamic side loads should be combined with the static loads due to quasi-static side loads directly to get the total loads for structural integrity evaluation. Since the maximum quasi-static side load is $\sim 37,000 \mathrm{lbs}$, by applying it on the SSME engine system model at the pressure center, the maximum static loads were obtained. Since the quasi-static side loads are the lateral loads that will excite the entire engine system, they will induce higher loads at the components that support the engine, e.g. the actuators, gimbal bearing, and the throat. Since the $3 \sigma$ dynamic load for the actuators was given in table 1 , it will be combined with the static load due to maximum quasi-static side load, i.e. $37,000 \mathrm{lbs}$, to obtain the maximum total loads. Therefore, during engine start the maximum transient load for the actuator is $\sim 200,000 \mathrm{lbs}$, i.e. $64,000 \mathrm{lbs}(3 \sigma$ dynamic load due to asymmetric side load transients) $+137,000$ lbs(static load due to maximum quasi-static side load). 


\section{Conclusions/recommendations}

The rocket engine side load transient is a very complicated problem, because it is a random process. To the author's knowledge, this is the first time that the mechanisms that cause the dynamic asymmetric and symmetric side loads have been identified, and a systematic approach was developed to solve the problems. A proprietary computer code was developed to generate the input forcing functions for the asymmetric dynamic side load transients, and by using the sub-scale nozzle test data the input forcing function was created for the symmetric side load transients. It is a practical approach that will generate the dynamic loads and the shock environments for rocket engine components in the early stage of new enginc developinent or engine nozzie modifications. As shown in Table 1, this approach yields excellent results for the SSME engine system. Since in the process of developing the methodology many assumptions have been made, the assumptions may create some issues. Therefore, in order to make it an analysis tool for generic engine systems these issues must be resolved. The following are the issues:

1. The scaling factor for the asymmetric side loads.

2. The characteristics, i.e. the frequency, the amplitude, and the number, of the pressure pulses for the symmetric side loads

3. What triggers the flutter that excites the nozzle local modes and then locks it into the $\mathrm{N}=2$ mode?

In order to resolve the issues, it is recommended to perform some tests to build a common data base that can be used for all the engines. The issues 1 and 2 can be resolved by subscale nozzle cold air flow tests, while full scale nozzle hot- fire tests should be performed to resolve the issue 3 .

Before the issues can be resolved, it is recommended to use the results from the SSME to analyze other engine systems. For the asymmetric side load transients, the scaling factor will be assumed to be .13 . As for the symmetric side load transients, only the pressure pulse frequency should be determined by tests or CFD analysis. As an example, the methodology was used to analyze the new RS-68 engine system developed by the Rocketdyne for the Boeing Delta IV launch vehicles. Due to very limited strain gage measurements on the actuators during the engine hot-fire testings, only asymmetric side load transients were analyzed. The measured actuator loads were within the range of the predictions.

\section{Acknowledgement}

This work was performed at the Rocketdyne Division, the Boeing Company. The material presented here is based on the work performed for a Boeing's IAD (internal application development) task. The author wishes to acknowledge the support of the Rocketdyne management.

\section{References}

1. Larson, E. W., and Ratekin, G. H., "Structural response to the SSME fuel feedline to unsteady shock oscillations", Part 2, The Shock and Vibration Bulletin, May 1982, pp177-182

2. Terhardt, M. , Gagermann, G. and Frey, M., "Flow separation and side-load behavior of truncated ideal rocket nozzles", AIAA 2001-3686, July, 2001

3. Watanabe, Y., Sakazume, N., and Tsuboi, M., "LE-7A engine nozzle problems during the transient operations", AIAA 2002-3841, July 2002

4. Nave, L. H. ,and. Coffey, G. .A, "Sea level side loads in high-area-ratio rocket engine", AIAA 73-1284, Nov. 1973

5. Richards, E. J., and Mead, D. J., "Noise and acoustic fatigue in aeronautics", John Wiley \& Sons, 1968, Chap. 8

6. Barrett, R. E., "Techniques for predicting localized vibratory environments of rocket vehicles", MSFC, NASA TN D-1863, Oct. 1963 